\title{
Data Dissemination based on Mobile Agent in Wireless Sensor Networks
}

\author{
Min Chen, Taekyoung Kwon, Yanghee Choi \\ School of Computer Science and Engineering \\ Seoul National University, Seoul, 151-744, Korea \\ mchen@mmlab.snu.ac.kr,\{tkkwon,yhchoi\}@snu.ac.kr
}

\begin{abstract}
Recently, mobile agents have been proposed for efficient data dissemination in sensor networks [1-6]. In the traditional client/server-based computing architecture, data at multiple sources is transferred to a destination; whereas, a task-specific executable code traverses the relevant sources to gather data in the mobile-agent based computing paradigm. As described in [1], many inherent advantages (e.g. scalability, extensibility, energy awareness, reliability) of the mobile agent architecture make it more suitable for sensor networks than the client/server architecture. In this paper, a mobile agent is exploited in three levels (e.g. node level, task level, and combined task level) to reduce the information redundancy and communication overhead.
\end{abstract}

\section{Introduction}

Most energy-efficient proposals are based on the traditional client/server computing model, where each sensor node sends its sensory data to a back-end processing center or a sink node. Because the link bandwidth of a wireless sensor network (WSN) is typically much lower than that of a wired network, a sensor network's data traffic may exceed the network capacity. To solve the problem of the overwhelming data traffic, $[1,2]$ proposed the mobile agent based distributed sensor network (MADSN) for scalable and energy-efficient data aggregation. By transmitting the software code, called "mobile agent (MA)", to sensor nodes, the large amount of sensory data can be reduced or transformed into small data by eliminating the redundancy. However, MADSN operates based on the following assumptions: (1) the sensor network architecture is clustering based; (2) source nodes are within one hop from a clusterhead; (3) much redundancy exists among the sensory data which can be fused into a single data packet with a fixed size. These assumptions pose much limitation on the range of applications which can be supported by MADSN.

Thus, we will consider MA in multi-hop environments with the absence of a clusterhead. In this paper, an MA is exploited in three levels to reduce the information redundancy and communication overhead for wireless sensor networks. Specifically, the MA is performing the following functions: (1) eliminating data redundancy among sensors by application context-aware local processing in node level; (2) eliminating spatial redundancy among closely-located sensors by data aggregation in task level; (3) reducing communication overhead by data concatenating in combined task level.

\section{The Architecture}

In the architecture of Fig. 1, sink queries multiple targets simultaneously by means of the MA. The data in different target regions is collected one by one.

In traditional scenarios, multiple different requests for physical information arrive at different times. We believe that there will come the application of multiple different tasks requested to be executed concurrently.

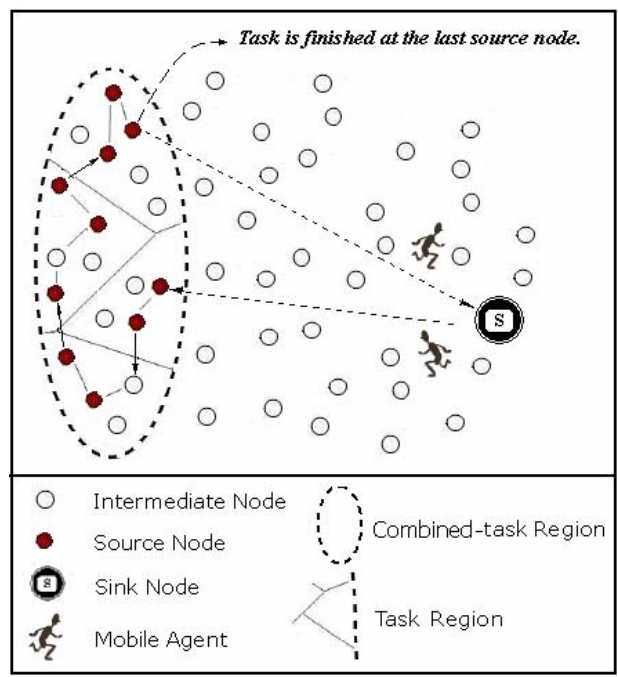

Figure. 1 A Simple Architecture of MA Based WSN

The reaction to a single task can range from a simple return of a result by collaborative processing among some sensor nodes (e.g. in the application to obtain the population of the objects, the system aggregates reports of individual objects right at the point of data source and sends the already-aggregated object counts), to a complex return of large size sensed data (e.g. a picture captured by an image sensor).

In wireless communications, the communication cost of sending a longer message is less than sending the same amount of data using many short messages. For the concurrent tasks associated with small amount of data, we can perform them by a single packet carrying multiple requests (e.g. one request for one task) and also concatenate their results into a single packet to save communication overhead. In Fig. 1, the combined multiple tasks will be executed one by one, so that the overall processing will take longer time. If the application's minimum quality of service (e.g. latency requirement) is not violated, especially in the case that the target region is far from a sink 
node, the energy efficiency of this combined executing can be significant.

We believe a combined-task query model that enables applications to initiate multiple tasks is needed to support this new and growing class of applications for sensor networks. Basically, the combined-task has three features: (1) all the tasks belonging to a combined-task can be processed by the common processing code part of the MA packet, thus extra communication overhead is not needed for the MA to carry additional processing code; (2) compared with the distance between the center of combined-task region and the sink, the task regions are likely to be close to each other; (3) all the tasks are requested concurrently by application.

\begin{tabular}{|c|c|c|c|}
\hline \multirow{2}{*}{$\begin{array}{l}\text { Combined task } \\
\text { and its sub tasks }\end{array}$} & \multicolumn{3}{|c|}{ Combined-task 1} \\
\hline & Task 1 & Task 2 & Task 3 \\
\hline Resultam Dara & \multicolumn{3}{|c|}{ Maximutn temperature } \\
\hline Interval & $200 \mathrm{~ms}$ & $200 \mathrm{~ms}$ & $400 \mathrm{~ms}$ \\
\hline duration & $1 \mathrm{~h}$ & $1 \mathrm{~h}$ & $1 \mathrm{~h}$ \\
\hline nectangle & {$[0,100,200,400]$} & {$[100,200,300,500]$} & {$[0,100,300,500]$} \\
\hline \multirow{2}{*}{$\begin{array}{l}\text { Combined task } \\
\text { and its sub tasks }\end{array}$} & \multicolumn{3}{|c|}{ Combined-task 2} \\
\hline & Task 1 & Task 2 & Task 3 \\
\hline Resultant Data & \multicolumn{3}{|c|}{ Population of the objects } \\
\hline Intenal & $5 s$ & $5 s$ & $10 \mathrm{~s}$ \\
\hline duration & $4 \mathrm{~h}$ & $3 \mathrm{~h}$ & $2 \mathrm{~h}$ \\
\hline nectangle & {$[0,100,200,400]$} & {$[100,200,300,500]$} & {$[0,100,300,500]$} \\
\hline
\end{tabular}

Figure. 2 Two Examples of Combined-task

Fig. 2 gives two examples of the combined-task query. Combined-task 1 and 2 belong to different applications which use different MA, since the resultant data are different. Three different tasks in both examples are requested concurrently, but the duration maybe different. In the last hour of combined-task 2, task 2 and task 3 already quit the processing, thus the combined-task is reduced to a traditional task. The rectangle of different tasks maybe overlapped, which means the sensed data generated by the sensors in the overlapped region will be used to contribute multiple tasks.

\section{Information Redundancy and Communication Overhead Reducing}

The proposed dissemination framework employs the MA's ability of carried processing code that allow the computation and communication resources at the sensor nodes to be efficiently harnessed in an application specific fashion. The MAs should adjust their behaviors depending on quality of service needs (e.g. data delivery latency) and the network characteristics to increase network lifetime while still meeting those quality of service needs. In this section, MA is dedicated to reduce the information redundancy and communication overhead in three levels so as to prolong network lifetime.

1) Node Level: Application Redundancy Eliminating by MAAssisted local Processing

With the development of WSN, "one deployment multiple applications" is a trend due to the application-specific nature of sensor networks. Such a trend must require sensor nodes to have various capabilities to handle multiple applications, which is economically infeasible. In general, using memory-constrained embedded sensors to store every possible application in their local memory is impossible. Thus, a way of dynamically deploying a new application is needed. In Fig. 1, the sink can assign the processing code (behavior) of MA based on the requirement of a specific application. The processing code carried by the MA packet only requires source nodes of local processing on the raw data as requested by the application. This capability enables a reduction in the amount of data transmission by allowing that only relevant information be extracted and transmitted.

2) Task Level: Spatial Redundancy Elimination by MAAssisted Data Aggregation

The degree of sensed data correlation among sensors is closely related to the distance between sensors, so that it is very likely for closely-located sensors to generate redundant sensed data. The MA aggregates individual sensed data when it visits each target source. Though this kind of aggregation technique is typically used in clustering or aggregation tree based data dissemination protocols, the MA assisted aggregation does not need any overhead to construct these special structures.

3) Combined Task Level: Communication Overhead Saved by MA Assisted Multiple Tasks' Data Concatenation

Instead of MA assisted aggregating data of individual sensor nodes right at the point of data sources, we propose the packet unification technique that unifies the several short data packets to one longer packet in order to reduce the communication overhead in combined-task level. Due to data concatenation, the duty cycle and communication overhead of intermediate sensor nodes can be reduced so as to increase network lifetime. However, such energy savings can be achieved usually at the cost of the prolonged data latency. To reduce latency, multiple tasks should be executed simultaneously by replicated MAs in the combined-task region. This decreases the overall execution time. Since replicating MAs incurs additional overhead, we should design carefully how many MAs should be dispatched in combined-task region depending on application's requirements.

\section{References}

[1] Hairong Qi, Yingyue Xu, Xiaoling Wang, "Mobile-Agent-Based Collaborative Signal and Information Processing in Sensor Networks," Proceeding of the IEEE, Vol. 91, NO. 8, pp.11721183,Aug. 2003

[2] Hairong Qi, Iyengar, S., Chakrabarty, K., "Multiresolution data integration using mobile agents in distributed sensor networks", IEEE Transactions on Systems, Man and Cybernetics, Vol. 31 , No. 3 , pp. 383 - 391, Aug. 2001

[3] Wu, Q., Rao, N.S.V., Barhen, J., etc. "On computing mobile agent routes for data fusion in distributed sensor networks", IEEE Transactions on Knowledge and Data Engineering, , Vol.16, NO. 6 , pp. $740-753$, June 2004

[4] Yu-Chee Tseng, Sheng-Po Kuo, Hung-Wei Lee and Chi-Fu Huang, "Location Tracking in a Wireless Sensor Network by Mobile Agents and Its Data Fusion Strategies", The Computer Journal,Volume 47, NO.4, July 2004: pp. 448-460

[5] Chee-Yee Chong, Kumar, S.P., "Sensor networks: evolution, opportunities, and challenges", Proceeding of the IEEE, Vol. 91, NO. 8, pp. 1247- 1256, Aug. 2003

[6] Arkady Zaslavsky, "Mobile Agents: Can They Assist with Context Awareness? ”, 2004 IEEE MDM'04, Jan. 2004 ,Berkeley, California 